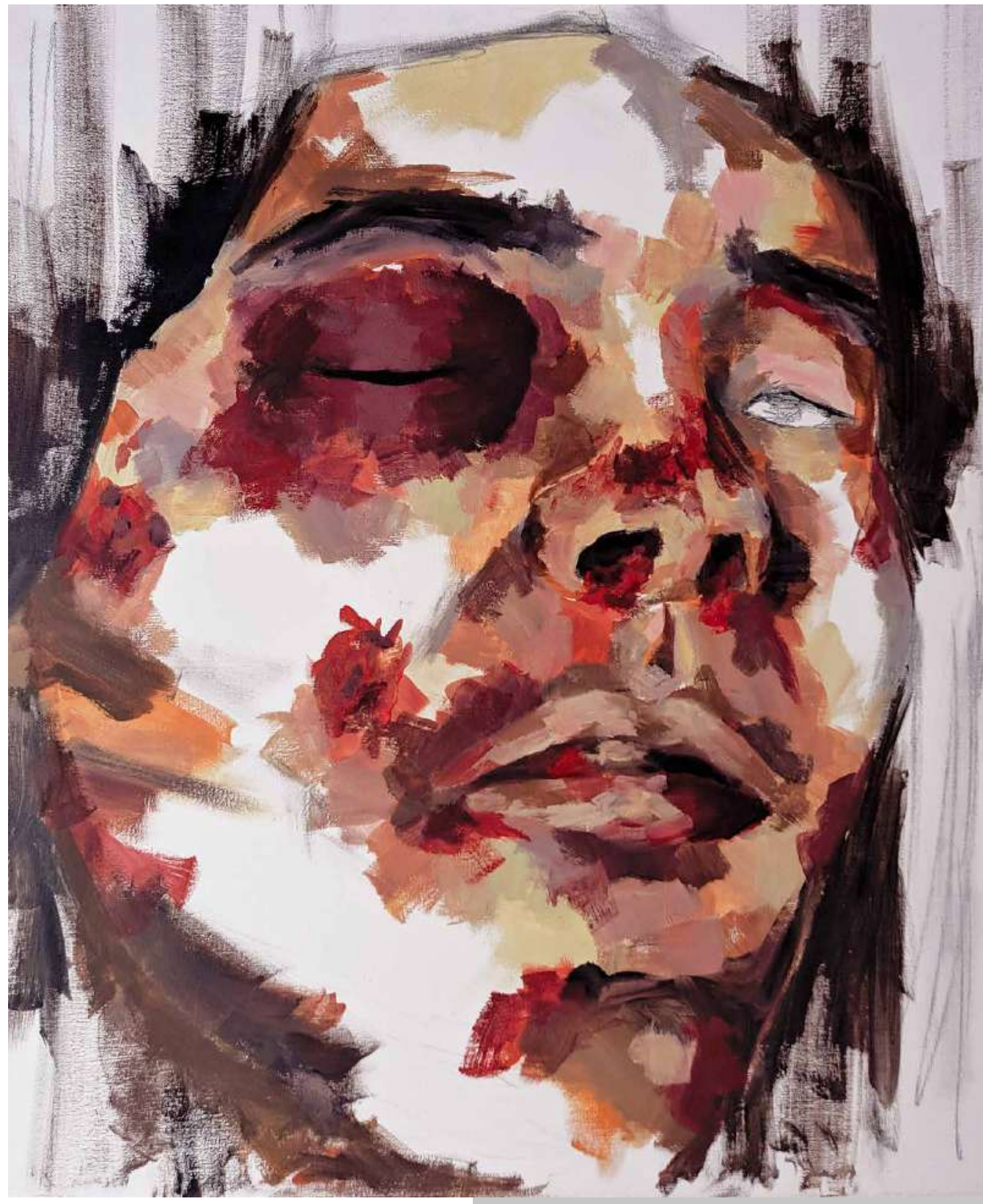

Artista invitada

Maria José Londoño

Aflicción

Óleo sobre lienzo

2019

Cortesía revista Ojo de Pez 


\title{
Repensando la nueva dinámica sociopolítica en Argentina y Brasil, 2011-2016*
}

\author{
Ana Natalucci (Argentina)** \\ Juan Pablo Ferrero (Argentina) ${ }^{* * *}$
}

\section{Resumen}

Este artículo analiza la dinámica sociopolítica durante la crisis del giro a la izquierda en Argentina y Brasil. ¿Por qué esa dinámica sociopolítica adoptó la forma de una alternancia institucional de poder en Argentina y un carácter antiinstitucional en Brasil? En el artículo se sigue una estrategia metodológica mixta: genealógica para comprender la disputa política, reconstrucción de las narrativas emergentes durante el ciclo de movilización y descriptiva para identificar las organizaciones movilizadas, sus demandas y repertorios de acción. El argumento que se sigue es que la estrategia movimentista del kirchnerismo contrastaba con la de desmovilización del Partido de los Trabajadores (PT). Mientras que la primera contribuía a canalizar los altos niveles de movilización al sistema político, la segunda fue decisiva en la

[326] crisis de un largo ciclo de representación política.

\section{Palabras clave}

Régimen Político; Política Comparada; Izquierda Política; Movilización Social; Kirchnerismo; Lulismo.

\footnotetext{
* Este artículo retoma los principales resultados de la investigación The End of the Left Turn in Latin America? New Actors and Discourses Shaping the Political Arena of the Post-Transition, financiada por el Departamento de Politica, Lenguaje y Estudios Internacionales y por la Oficina de Relaciones Internacionales, Universidad de Bath, Reino Unido, 2019. La investigación se realizó entre 2015 y 2019 bajo la dirección del Dr. Juan Pablo Ferrero y la coordinación de la Dra. Ana Natalucci en Argentina y de la Dra. Luciana Tatagiba en Brasil.

** Licenciada en Comunicación Social. Magíster en Investigación en Ciencias Sociales. Doctora en Ciencias Sociales. Investigadora adjunta del Consejo Nacional de Investigaciones Científicas y Técnicas (Conicet), investigadora del Centro de Innovación de los Trabajadores, Universidad Metropolitana para la Educación y el Trabajo (CITRA/UMET), profesora adjunta de la Universidad de Buenos Aires, Argentina. Correo electrónico: anatalucci@gmail.com - Orcid: 0000-0001-5362-2098 Google Scholar: https://scholar.google.com/citations?hl=es\&user=NxcAu9oAAAAJ

*** Licenciado en Ciencia Política. Doctor en Ciencias Sociales y Políticas. Profesor titular en el Departamento de Política, Lenguaje y Estudios Internacionales, Universidad de Bath, Reino Unido. Coeditor del Bulletin of Latin American Research (BLAR). Correo electrónico: J.P.Ferrero@bath. ac.uk - Orcid: 0000-0002-9713-7534
} 


\title{
Cómo citar este artículo
}

Natalucci, Ana y Ferrero, Juan Pablo. (2021). Repensando la nueva dinámica sociopolítica en Argentina y Brasil, 2011-2016. Estudios Políticos (Universidad de Antioquia), 60, pp. 326-349. DOI: 10.17533/udea.espo.n60a14

\section{Rethinking the New Socio-Political Dynamic in Argentina and Brazil, 2011-2016}

\begin{abstract}
This article analyzes the socio-political dynamics during the left turn crisis in Argentina and Brazil. Why did this socio-political dynamic take the form of an institutional alternation of power in Argentina and an anti-institutional character in Brazil? We follow a mixed methodological strategy: genealogical, to understand the political dispute and reconstruct the emerging narratives during the mobilization cycle; and descriptive, to identify the mobilized organizations, their demands, and repertoires of action. The argument is that the Kirchnerism movement's strategy in Argentina contrasts with the demobilization strategy of the Workers' Party (PT) in Brazil. While the former contributed to channeling high levels of mobilization to the political system, the latter was decisive in the crisis of a long cycle of political representation.
\end{abstract}

\section{Keywords}

Political Regime; Comparative Politics; Political Left; Social Mobilization; Kirchnerism; Lulism. 


\section{Introducción}

A principios del siglo xxı, en algunos países de América del Sur se produjo un giro a la izquierda con un considerable apoyo electoral (Levitsky y Roberts, 2011; De la Torre, 2013; Silva, 2017; Etchemendy, 2019), entre ellos, Venezuela, Brasil, Argentina, Bolivia, Uruguay y Ecuador. Este giro tuvo dos características centrales. Por un lado, la construcción de una frontera con el neoliberalismo a partir de la idea del «regreso del Estado»; y por el otro, la constitución de una dinámica sociopolítica con un nuevo tipo de vínculo entre gobiernos, sindicatos y organizaciones sociales. Durante este giro, los gobiernos siguieron tres premisas: a) un pacto de consumo para el crecimiento de la demanda y fortalecimiento del mercado interno, b) un pacto de inclusión social orientado a la expansión de los sistemas de protección social y c) un pacto de soberanía de consolidación de espacios regionales (Stefanoni, 2016). Estos pactos mantuvieron su vigencia por lo menos hasta 2009, posibilitados por el crecimiento económico y el «consenso de los commodities» (Svampa, 2013). En términos generales, el programa neodesarrollista generó una situación de win-win, donde todos los sectores - trabajadores formales, informales, sectores medios y empresariales - se beneficiaron, siendo así decisiva para la creación del nuevo consenso político en torno a coaliciones de intereses (Zucco, 2008; Sidicaro, 2011; Singer, 2012).

En Argentina y Brasil este ciclo se inició en 2003, a partir de la asunción de Luiz Inácio Lula da Silva (2003-2006, 2007-2010) en enero y de Néstor Kirchner (2003-2007) en mayo. Cada uno tuvo diferentes modos de llegar a la presidencia. En principio, el Partido de los Trabajadores (PT) y el Partido Justicialista (PJ) tuvieron roles diferentes en las reformas neoliberales. Mientras el PT se erigió como un espacio de resistencia, el PJ fue protagonista en los gobiernos de Carlos Menem (1989-1995, 19951999). Esta diferencia permite explicar por qué la victoria presidencial de da Silva se expresó en términos de un «punto de arribo» (Ferrero, 2014) de las fuerzas popular-democráticas, en un largo camino iniciado con el fin de la dictadura y la transición a la democracia. Por el contrario, Kirchner accedió a la Presidencia con un bajo nivel electoral (22,3\%), en un contexto de profunda crisis de representación y fragmentación partidaria, luego de la crisis de 2001 que lo llevó a construir un electorado poselectoral (Cheresky, 2006). 
Esta diferencia originaria es fundamental para comprender las relaciones entabladas entre cada gobierno y el campo multiorganizacional, entendido como el espacio social donde confluyen organizaciones con identidades y demandas particulares. Respecto de este vínculo, el lulismo impulsó un «reformismo desmovilizador», un intento deliberado de deconstruir antagonismos y desalentar la movilización callejera a favor de la participación institucional y la reforma dentro del aparato estatal (Singer, 2012; Tatagiba, 2019). Esta estrategia de integración con desmovilización fue eficaz mientras el gobierno contara con un alto nivel de aprobación de la opinión pública; empero, en los primeros meses de la segunda presidencia de Dilma Rousseff (2011-2014, 2015-2016) la irrupción de las protestas anticorrupción hundió la popularidad del PT. Contrariamente, el kirchnerismo instituyó una gramática movimentista de acción colectiva que le permitió consolidar la élite gubernamental y un movimiento popular con capacidad de movilización e integración al Estado (Sidicaro, 2011; Natalucci, 2019) que con matices sostuvo hacia el fin del ciclo.

Así, el punto de inflexión se produjo con las presidencias de Cristina Fernández de Kirchner (2007-2011, 2011-2015) en Argentina —sobre todo, en el segundo periodo-y de Dilma Rousseff en Brasil (2011-2015). Aunque aparentemente ambas implicaban una continuidad, mostraron cambios significativos respecto de sus fuerzas políticas en la reconfiguración de la relación entre el gobierno y el espacio multiorganizacional, y en el modo en que se procesaron las demandas en el contexto de la crisis internacional de 2008-2009. Fernández de Kirchner, que mantuvo una estrategia heterodoxa en materia económica, en el plano político tendió a desarticular la gramática movimentista minimizando el rol de las mediaciones político-organizativas, al mismo tiempo que mantuvo la movilización de sus aliados. Rousseff implementó medidas económicas ortodoxas frente a la crisis, perjudicando a su base de apoyo y manteniendo la estrategia de desmovilización impulsada por Da Silva. En este sentido, la crisis internacional fue clave al aparejar la ruptura de la situación de win-win y abrir el debate en torno a la profundización o moderación de las políticas redistributivas. La respuesta política de cada gobierno contribuyó a debilitar las alianzas entre las fuerzas sociales y las coaliciones gubernamentales. En el caso de Brasil, se vio agravado por la situación de un Congreso hiperfragmentado, con partidos sin anclaje social y, por lo tanto, con incentivos para desertar de la coalición gobernante, lo cual aceleró el impacto institucional de la movilización callejera anticorrupción al disolver los frágiles acuerdos parlamentarios que garantizaban la gobernabilidad. 
El objetivo de este artículo es analizar esa dinámica sociopolítica en Argentina y Brasil en el contexto de la crisis del giro a la izquierda. Si bien en esta crisis podía percibirse una sensación general de agotamiento del kirchnerismo y el lulismo, aún falta indagar por qué la nueva dinámica sociopolítica adoptó la forma de una alternancia institucional de poder en Argentina y un carácter antiinstitucional en Brasil. Nuestro argumento es que en Argentina el campo multiorganizacional mantuvo una relativa autonomía que le permitió tener un dinamismo particular y, al mismo tiempo, canalizar vía la movilización los altos niveles de disconformidad social, esta característica fue clave para que el kirchnerismo perdiera el monopolio de la movilización, más no la calle. En Brasil, el PT se asoció con sectores ortodoxos en el plano económico mientras mantuvo su estrategia de desmovilización de sus aliados, ambos aspectos — sumados al Congreso fragmentado y partidos parlamentarios sin apoyo popular - fueron claves para reforzar el clima «anti-PT» y contribuir a la generación de la crisis de representación política.

Durante la investigación se presentaron dificultades metodológicas respecto del carácter comparado que requería analizar el proceso de ambos países que, sin duda, tenían algunas coincidencias, pero también contrastes significativos. ${ }^{1}$ Para resolver este dilema se definieron tres dimensiones analíticas desagregadas de la noción de dinámica sociopolítica: ciclos de movilización, imaginarios y representación política. Para la construcción de los datos se utilizaron dos tipos de fuentes: a) una base cuantitativa comparada de eventos de protestas sociales, construida a partir de los periódicos La Nación -10 de diciembre de 2011 al 9 de diciembre de 2015, Argentina_y el Folha de San Pablo — 1. o de enero de 2011 al 16 de agosto de 2016, Brasil-; y b) un exhaustivo trabajo de campo cualitativo conformado de cuarenta entrevistas en profundidad, veinte para cada país, a dirigentes políticos, de organizaciones territoriales, sindicales y juveniles. La complementariedad de datos cuantitativos y cualitativos ha permitido elaborar un mapa de la dinámica de la movilización social, a la vez que profundizar sobre los sentidos, imaginarios políticos y consecuencias para el sistema político.

\footnotetext{
${ }^{1}$ Por limitaciones de espacio y para no extenderse en demasía sobre las coincidencias y contrastes, véase Juan Pablo Ferrero, Ana Natalucci y Luciana Tatagiba (2019). Sobre cuestiones generales de las investigaciones comparadas, véase Torcuato Di Tella (1998).
} 


\section{Hacia una noción multidimensional de la dinámica sociopolítica}

Como ya se mencionó, el objetivo del artículo es analizar la dinámica sociopolítica configurada durante la crisis del giro a la izquierda, para lo cual se elaboró una propuesta teórica que permita captar la multidimensionalidad de los procesos en juego. El carácter ecléctico de esta propuesta queda justificado por la complejidad de los procesos, actores y acontecimientos implicados en el ciclo analizado. Por supuesto que esta conceptualización no agota todo lo que pueda decirse de estos años, pero sin duda constituye un registro explicativo de alta productividad analítica.

El concepto de «dinámica sociopolítica» implica al conjunto de reglas formales e informales del juego político situado históricamente que, con diferentes niveles de sedimentación, organiza las interacciones de los agentes individuales y colectivos, incluyendo sus dimensiones estructurales, de acción y efectos en el régimen político. Esta conceptualización supone un desplazamiento de las perspectivas «organizaciones céntricas» que consideran a los movimientos como actores independientes, como también de las perspectivas que enfatizan sobre las estrategias gubernamentales; más bien, aquí se propone concentrarse en las interacciones conflictivas entre organizaciones y gobiernos, en esas «intersecciones» (Banaszak, 2005) que configuran un tipo particular de dinámica sociopolítica.

El análisis de la crisis del giro a la izquierda desde el concepto de dinámica sociopolítica permite dar cuenta de cambios significativos, sin perder de vista que estos se inscriben en un contexto regional y global más amplio, signado por grandes (dis)continuidades en las relaciones Estado-sociedad que incluyen la mayor fragmentación social, la crisis de la representación política y de identidades tradicionales, entre otras.

\subsection{Dimensiones de la dinámica sociopolítica}

En la definición clásica de Sidney Tarrow (1997) el ciclo de movilización implica un proceso de intensificación de los eventos de protestas, en el que se produce una difusión de los repertorios de acción, la incorporación de organizaciones o actores sociales ya consolidados, junto con la emergencia 
de nuevos colectivos. Una de las principales críticas a esta perspectiva ha sido la disyuntiva que plantea entre insiders y outsiders del sistema, disociando el espacio social y del político-institucional, y circunscribiendo a los movimientos al primero. De hecho, al corriente de esta crítica, Doug McAdam, Sidney Tarrow y Charles Tilly (2005) han propuesto un modelo dinámico a partir de la noción de «contienda política», entendida como la «interacción episódica, pública y colectiva entre los reivindicadores y sus objetos» (p. 5), mientras uno de los reivindicadores sea un gobierno y cuando la satisfacción de esas reivindicaciones afecte los intereses de alguno de los intervinientes. Pese al esfuerzo de los autores, lo cierto es que mantienen la premisa de los movimientos como outsiders, resaltando la idea de contención en el sentido de controversia o confrontación.

Evidentemente, en el caso analizado esa disyuntiva fue un problema, en tanto las relaciones entre el campo político y los actores del campo multiorganizacional habían dejado de ser principalmente de confrontación para asumir formas de alianzas estratégicas, compromisos y negociaciones, de ahí que se retome el planteo de Daniel Cefaï (2011), para quien la movilización supone un proceso por el cual las organizaciones intentan definir una situación problemática, entablando relaciones de cooperación y de conflicto, incluso con los gobiernos. Los movimientos sociales y sus organizaciones crean espacios de experiencias, no solo siguiendo una racionalidad instrumental-estratégica, sino también guiados por lealtades, apreciaciones estéticas, cuestiones éticas o preferencias políticas. Esta perspectiva cuestiona la idea de racionalidad única y propone adoptar la de «regímenes de compromiso» (p. 139), en los cuales no hay intereses que se agregan, sino demandas que se construyen en contextos particulares.

En un proceso de movilización siempre hay un objeto de disputa, algo sobre lo que se delibera, se propone, se cuestiona. En algunos casos ese objeto se configura bajo la forma de demanda y en otros como un problema público. Este se inscribe en diferentes memorias, narrativas e imaginarios, y alude a una dimensión del orden de la controversia, de algo pendiente por hacer y sobre quien recae la responsabilidad de hacerlo (Pereyra, 2013). Durante un proceso de movilización la generalización de una demanda bajo la forma de un problema público es importante para definir su naturaleza y los posibles efectos que puede implicar para el sistema político. 
Respecto del tipo de movilización y sus posibles efectos, la propuesta de Gino Germani (2003) resulta más operativa, considerando las particularidades de los regímenes políticos de los casos estudiados. Retomando su clasificación, se puede diferenciar entre movilización integrada y no integrada. Mientras la primera se desarrolla dentro de las reglas vigentes del juego político, la movilización no integrada implica una disputa parcial o completa sobre las reglas que regulan el sistema político. En ambos casos, las reglas que se siguen o disputan aluden a las formas de participación, mecanismos de representación y dispositivos de legitimidad.

Ahora bien, en un ciclo de movilización se van configurando diferentes imaginarios, en los casos estudiados: «populismo», «república» y «corruptos». Siguiendo a Bronislaw Baczko (1984), esta noción alude a la dimensión simbólica de la acción social, a la representación de la realidad resultante de las interacciones que tienden a construir una realidad dominante, una imagen general de la sociedad que influye en los comportamientos individuales y colectivos. Dichas interacciones constituyen condiciones simbólicas de posibilidad de acción. Los imaginarios sociales constituyen «las formas en que las personas imaginan su existencia social, cómo funcionan las cosas entre ellos y sus compañeros, las expectativas que normalmente se cumplen y las imágenes que subyacen a estas expectativas» (Ferrero, 2016, p. 56. Traducción propia). En este sentido, Baczko (1984) sostiene que los imaginarios sociales son fundamentales para la dimensión temporal de la acción colectiva, en tanto implican una doble proyección de significado, tanto en el pasado como en el futuro, entre las memorias y las utopías; asimismo, establecen los límites del campo de acción desempeñando un papel crucial para la acción colectiva y legitimando ciertas articulaciones y coordinaciones por sobre otras. En definitiva, los imaginarios sociales permiten la captura de significados múltiples e inestables de la realidad social elaborados a partir de las tradiciones, recreadas por las experiencias de acción colectiva.

Finalmente, la representación constituye la última noción central para la perspectiva multidimensional de la dinámica sociopolítica. Siguiendo a Hanna Pitkin (1985), la representación implica «hacer presente en algún sentido algo que, sin embargo, no está presente literariamente o de hecho» (p. 10). En esta clave, hay que comprenderla en términos de «que significa, bajo qué 
circunstancias es posible y cómo se justifica» (p. 10). En definitiva, alude a la posibilidad que los agentes se sientan representados y puedan ofrecer razones de esa representación.

El debate en torno a la representación es significativo para comprender los alcances de la crisis del giro a la izquierda, en tanto permite explicar no solo la emergencia de alternativas partidarias al kirchnerismo y al PT, sino también los modos en que se produjo el recambio de autoridades en cada país. De ahí que sea necesario analizar tanto las estrategias de participación - partidaria-no electoral, electoral-no partidaria y partidaria-electoralcomo las percepciones en torno al gobierno y al Estado, las cuales permiten reconocer las razones dadas por los actores sobre por qué deciden movilizarse y el horizonte sociopolítico en el que se puede inscribir dicha acción.

\section{Un nuevo ciclo de movilización: repensando Argentina y Brasil}

¿Cuáles fueron las características del ciclo de movilización activado en 2012 y de qué modo contribuyó a configurar la nueva dinámica sociopolítica? En principio, este ciclo generó las condiciones de posibilidad para la crisis del

[334 ] giro a la izquierda, sin embargo, no está claro que haya tenido la capacidad para recrear un nuevo consenso que sepultara electoral y políticamente de modo definitivo al kirchnerismo y al lulismo. La importancia de considerar estos datos es sustancial para entender la heterogeneización del campo multiorganizacional y la pérdida del monopolio de la calle por parte del kirchnerismo como la profundización de la estrategia de desmovilización del PT y la elaboración de ese imaginario «anti-PT».

Entre 2011 y 2015 en Argentina acontecieron 1593 eventos de protesta, con un promedio anual de 394; en Brasil se produjeron 1113 eventos, sin contar 172 correspondientes al periodo del impeachment, con un promedio de 222,6 protestas por año; es decir, que ese promedio anual en Argentina representa 77\% más que en Brasil. El pico en Argentina ocurrió en 2012 —490 protestas - luego de la reelección de Fernández de Kirchner, con una tendencia decreciente hacia 2015 —45 en 2013; 367 en 2014; 317 en 2015- En cambio, la dinámica de la protesta en Brasil fue más oscilante, con un pico en 2013 de 445 protestas. 
Gráfica 1. Eventos de protesta social en Argentina y Brasil, 2011-2016.

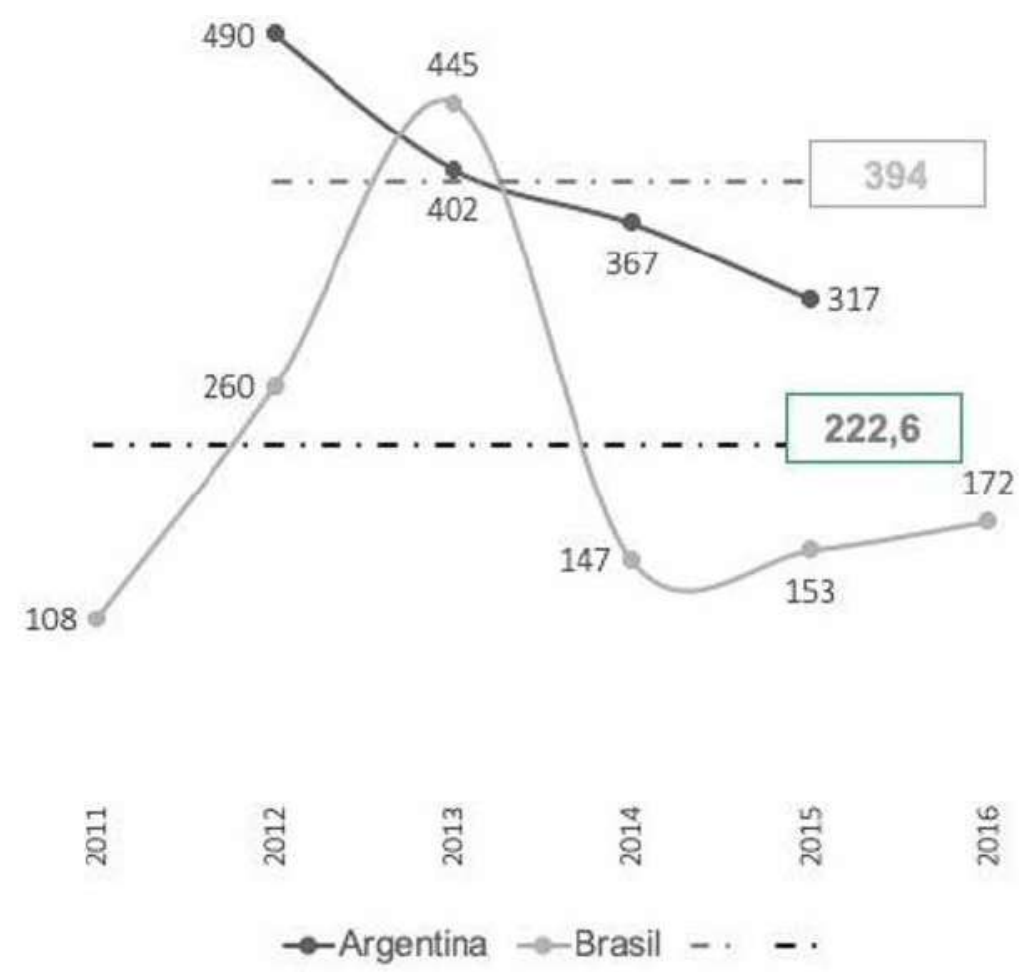

Fuente: tomado de Ferrero, Tatagiba y Natalucci (2018).

\section{La constitución de una nueva dinámica sociopolítica}

\subsection{La movilización integrada en Argentina}

El ciclo de movilización tuvo dos principales características: la primera, su lógica heterogénea en términos de los actores, demandas y repertorios; la segunda, un tipo de movilización integrada en términos de su relación con el gobierno y de su devenir dentro de las reglas instituidas del sistema político. Recapitulando, el ciclo se configuró en una coyuntura política y económica particular: la crisis internacional de 2008-2009, el fallecimiento del expresidente Kirchner en octubre de 2010 y la reelección de Fernández de Kirchner en octubre de 2011 con 54,11\% de los votos. La amalgama de estos eventos afectó la posición del kirchnerismo en el campo político. Por un lado, la estrategia neodesarrollista entró en crisis debido a la disminución del precio de los commodities, impactando negativamente en la capacidad distributiva del gobierno. Esta estrategia había sido decisiva para la innovación 
en materia de políticas sociales, particularmente aquellas orientadas a los sectores informales de la economía. Por otro lado, el fallecimiento de Kirchner aparejó que Fernández de Kirchner — dedicada principalmente a la gestión presidencial - asumiera el liderazgo de la fuerza política. Este cambio trajo consecuencias para la gramática movimentista, en tanto las organizaciones creadas por el kirchnerismo — como La Cámpora — fueran tomando un lugar preponderante en las instancias de toma de decisiones, desplazando a grupos fundadores como los sindicatos y organizaciones territoriales (Natalucci, 2019). Entre otras consecuencias, algunos grupos abandonaron el kirchnerismo, el cual vio debilitada su capacidad de articulación política, entendida como una de las condiciones para el sostenimiento del éxito electoral, la legitimidad y el apoyo popular. Un ejemplo paradigmático de este proceso fue la ruptura de la Confederación General del Trabajo (CGT) en dos facciones.

En esta coyuntura se abrió el ciclo de movilización en 2012. Cuando anteriormente se refirió el carácter heterogéneo, se aludió, entre otras cuestiones, a que no se observa un único grupo por el que se pueda explicar el incremento de la participación callejera: los sindicatos participaron de $37,3 \%$ en las protestas, los vecinos $14,2 \%$, los empresarios $8,6 \%$, partidos políticos $7,2 \%$, organizaciones de base territorial $6,5 \%$ y familiares y amigos de víctimas $5,7 \%$. Así, en las movilizaciones participaron grupos antikirchneristas — sectores medios, empresarios y líderes de la oposición partidaria—, grupos exkirchneristas — sindicatos y organizaciones sociales—, grupos kirchneristas — sindicatos, organizaciones sociales y trabajadores informales- y colectivos ajenos a la dicotomía kirchnerismo-antikirchnerismo — mujeres y familiares y amigos de víctimas-.

Los principales formatos fueron las manifestaciones $(28,9 \%)$ y los cortes de calle $(28 \%)$, las huelgas $(16,7 \%)$ y las solicitadas-conferencias de prensa $(7,1 \%)$. Estos formatos tienen una modularidad significativa, en el sentido que suelen ser usados por diferentes tipos de organizaciones, y al mismo tiempo un bajo nivel de beligerancia.

En relación con el gobierno, mientras algunos grupos mantenían una fuerte oposición, otros destacaban su rol mediador. Retomando la diferenciación de Germani (2003) respecto de movilizaciones integradas y no-integradas, este ciclo fue integrado en el sentido de que los actores movilizados -incluso los antikirchneristas - no pretendían la renuncia de Fernández de Kirchner, sino que su objetivo era fortalecer alternativas 
opositoras. Al respecto, un ciberactivista relató que su objetivo era «ayudar a unir a la oposición» (comunicación personal, Buenos Aires, 26 de noviembre, 2015). En tal dirección, este ciclo fue decisivo para la creación de nuevos espacios político-partidarios, como Cambiemos, y su triunfo electoral en 2015, sin que esto llevara a la desestabilización del sistema político.

\subsection{Imaginarios en pugna}

El triunfo de Fernández de Kirchner en 2011 fue acompañado de una radicalización discursiva de la élite kirchnerista. Un claro ejemplo ha sido la consigna Cristina Eterna, que expresaba la intención de reformar la constitución para permitirle el acceso a un tercer mandato. Por su parte, Fernández de Kirchner mencionaba recurrentemente la idea de Vamos por todo, la cual dejaba entrever su decisión de avanzar sobre los cambios que faltaban a los iniciados en 2003. Esta radicalización alarmó a los grupos antikirchneristas que ante la posibilidad de una reforma constitucional caracterizaron al kirchnerismo como un «jacobinismo estatista» (Vommaro, 2017, p. 31). Al mismo tiempo, esta percepción encontró eco en los empresarios que observaban la intervención del gobierno en la economía como un exceso, sobre todo por las restricciones en el mercado cambiario. La corrupción funcionaba como la explicación a estos excesos. ${ }^{2}$ Sobre estas percepciones se construyó un imaginario en torno a la «chavización» de Argentina (Vommaro, 2017), decisivo para que estos decidieran manifestarse en el espacio público. Es importante aclarar que estos sectores, inscriptos en la larga tradición antiperonista, tuvieron una corta participación en la vida pública durante la crisis de 2001, sin representación durante el kirchnerismo (Torre, 2017). En este marco, en 2012 se lanzaron a la calle reactivando un vocabulario de motivos liberal-republicano (Gold, 2019), cuya narrativa contenía las palabras «libre mercado», «competencia», «república», opuestas a lo que representaba el populismo kirchnerista.

En paralelo a esta disputa se produjeron tensiones al interior de la fuerza política oficialista respecto de quién era el poseedor de los votos. Algunos líderes peronistas — que luego formaron el Frente Renovador- y facciones de la CGT Azopardo cuestionaron la versión oficial que los votos

\footnotetext{
${ }^{2}$ Las denuncias de casos de corrupción desempeñaron un rol central en la constitución del polo antikirchnerista, en tanto empezó a configurarse como un punto débil del gobierno, no solo en términos de responsabilidades personales, sino de cuestionamiento a la política económica respecto de los subsidios estatales y contra la estrategia intervencionista del gobierno (Natalucci, 2018).
} 
pertenecían únicamente a Cristina, sino que cada uno había hecho su aporte electoral. Estos sectores cuestionaban la legitimidad de la presidenta en tanto ella no representaba todo el espacio político-partidario. En esta disputa se dirimía, en definitiva, el lugar que cada grupo tenía en los procesos de toma de decisiones. Como contraparte, Fernández de Kirchner inició un proceso de desarticulación del espacio kirchnerista, tal como había actuado hasta entonces, desplazando a un lugar secundario a los díscolos.

Los grupos kirchneristas también tenían críticas: sostenían que la radicalidad discursiva no se había traducido en cambios estructurales. Uno de sus ejemplos recurrentes era que el gobierno había decidido gestionar la crisis internacional, en vez de aprovechar la oportunidad para implementar reformas estructurales. Otro ejemplo apuntaba al límite distribucionista del modelo neodesarrollista. Las críticas de estos grupos eran particulares, probablemente porque su interés se orientaba a instalar demandas que habían emergido durante el giro a la izquierda.

Finalmente, en el ciclo se produjeron protestas impulsadas por vecinos, mujeres y familiares y amigos de víctimas. Todos estos colectivos no tenían conexiones y sus demandas eran diversas: los vecinos se manifestaban contra [338] el gobierno (6,3\%), por la seguridad $(17,7 \%)$ y hábitat, infraestructura y transporte $(9,6 \%)$. Esta última demanda fue significativa ya que los prolongados cortes en el suministro eléctrico y el deficiente funcionamiento del sistema de transporte en la zona metropolitana reforzaban el imaginario de corrupción instalado por los grupos antikirchneristas. Las mujeres Ilevaron adelante la multitudinaria movilización \#NiUnaMenos. Alrededor de este eje, el balance era equilibrado: el avance en derechos civiles como el matrimonio igualitario se compensaba con no haber despenalizado el aborto (Rey, 2019). Asimismo, los antagonistas identificados eran el Poder Judicial o el Legislativo antes que el Ejecutivo, por lo que sus actuaciones no desafiaron abiertamente al gobierno.

Como puede observarse, no hay un imaginario que haya monopolizado el ciclo de movilización, sino que se identificaron dos principales: uno relacionado con el autoritarismo y la naturaleza populista del gobierno que tenía como horizonte la Venezuela chavista, la difusión de la corrupción y la consecuente amenaza a los principios liberales y republicanos; y otro que afectaba al kirchnerismo, en tanto cuestionaba la representación de Fernández de Kirchner sobre todo el espacio, al mismo tiempo que identificaba las dificultades para resolver problemas estructurales. Estos dos 
imaginarios no lograron construir una movilización eminentemente opositora. Complementariamente, surgieron demandas y problemas públicos que rompían la dicotomía kirchnerismo-antikirchnerismo o populismo-república, en especial la movilización feminista que atravesaba a ambos espacios por igual. Para resumir, no solo había heterogeneidad respecto de los actores movilizados, sino también de las demandas e imaginarios en juego.

\subsection{Una crisis de representación de corto alcance}

Ahora bien, que estos imaginarios hayan permanecido en paralelo o incluso en competencia no implica que no hayan contribuido a debilitar el régimen de dominación política erigido por el kirchnerismo. Siguiendo a Luc Boltanski (2014), la noción de dominación implica las maneras de orientar un poder dado en nombre de una forma particular de política que requieren de justificaciones que lo legitimen socialmente. La crisis de representación que estalló en diciembre 2001 tuvo como consigna iQue se vayan todos! El kirchnerismo pudo construir una representación política ubicada en la centroizquierda del campo político, mientras daba respuestas a una amplia variedad de demandas. Por esta razón y por largo tiempo tuvo una posición dominante en el campo político, evidenciada en sus rotundos triunfos electorales de 2007 y 2011. Esta posición dominante se expresaba también en la convocatoria a movilizaciones plebiscitarias que reactivaban el sentimiento plebeyo de los sectores populares y reforzaban su pertenencia al movimiento nacionalpopular, de larga tradición en Argentina.

Esta situación cambió luego del ciclo de movilización en 2012, cuando el kirchnerismo perdió el monopolio de las calles por la emergencia de los grupos anti y exkirchneristas. Respecto de estos últimos, se escindió un sector de la CGT, mientras un grupo peronista fundó el Frente Renovador, aparejando que el kirchnerismo redujera su representación sobre el centro del espacio político. La emergencia de los grupos antikirchneristas no fue significativa en términos cuantitativos, más bien tuvo un impacto cualitativo. Integrados por ciberactivistas, partidos políticos de centro-derecha y derecha, y ciudadanos que en la dicotomía populismo-república se autoinscribían en esta última. Los ciberactivistas cumplieron un rol fundamental en tanto mediadores entre esos ciudadanos sin representación y los partidos políticos. Estos grupos tenían una concepción liberal sobre la representación política. Durante los cacerolazos de 2012-2013 reformularon la consigna de 2001 iQué se vayan todos! por iQue se vayan ellos!, en referencia al gobierno 
(Gold, 2019). Y el modo que tenían para que se fueran no era por medio de la renuncia de Fernández de Kirchner, sino por la construcción de un partido alternativo y competitivo para las elecciones de 2015. La idea que unificaba a estos grupos era que «la oposición se uniera para enfrentar al kirchnerismo». En este sentido, esta apuesta política no era antisistémica, por el contrario, requería de la estabilidad del campo político y de la continuidad de las reglas. Estos intentos tuvieron éxito, en 2015 Cambiemos ganó las elecciones en segunda vuelta.

En estas condiciones se produjo la alternancia institucional en 2015, con una nueva fuerza política con representación de la centro-derecha, mientras el kirchnerismo mantenía su representación solo sobre los sectores de centro-izquierda.

\section{La dinámica sociopolítica en Brasil}

\subsection{Movilización no-integrada}

Brasil experimentó un nuevo ciclo de movilización entre 2011 y 2015, comparable con los ciclos ocurridos durante el periodo de la posdictadura [340] (1984) y el proceso de destitución del presidente Collor de Mello en 1992 (Saad-Filho, 2013). Una de las mayores diferencias entre ese nuevo ciclo y los anteriores es el campo social heterogéneo debido a la incorporación de nuevos colectivos provenientes de los sectores medios urbanos, a los ya tradicionales sindicales y a organizaciones territoriales. La característica principal de este ciclo de movilización fue que sus participantes se distanciaron del PT y de la izquierda tradicional, adoptando una postura autónoma o directamente antagonizando con el PT, responsabilizándolo como la principal fuente de los males del país. Sin embargo, los casos de corrupción como el Lava Jato —el esquema de lavado de dinero realizado a través de Petrobras y en el que participaron políticos y empresarios de todo el espectro político- afectaron a toda la élite y, por lo tanto, las protestas sociales adoptaron una dinámica antisistémica que resistieron la integración dentro de las reglas del juego político.

En términos comparativos, los brasileños protestaron menos que los argentinos. Respecto de la evolución de las protestas, los eventos fueron in crescendo: de 108 en 2011, 260 en 2012 y el máximo de 445 en 2013, con las Ilamadas Protestas de junio. Como muestra la gráfica 1, la cantidad de 
protestas cayó significativamente (147) en 2014 cuando Rousseff accedió a su reelección con un estrecho margen de 3,2\%. La relación entre la movilización de 2013 y el resultado electoral de 2014 es objeto de controversia para sus protagonistas. Un miembro de la CUT ha sostenido que «fue construido por la élite brasileña [...]. Esperaban que los miembros del parlamento, la movilización masiva y la clase media alta podrían unirse para hacer que su candidato gane» (comunicación personal, São Paulo, 1. de febrero, 2016). Sin embargo, un miembro de Vem Pra Rua, una de las organizaciones protagonistas de las Protestas de junio, ofrece una explicación diferente: «Nunca he sido parte de ningún partido. Nunca he estado afiliado a algo. Creo que tengo un poco de odio por los partidos... ¿Activismo?, supongo que desde siempre [...]. Si [Dilma] no hubiera ganado, hubiéramos estado aplaudiendo como locos» (comunicación personal, São Paulo, 24 de noviembre, 2016). Ambos extractos muestran que, si bien las protestas adquirieron un tono marcadamente antiPT, no estaban necesariamente a favor del Partido de la Social Democracia Brasileña (PSDB) o de otra fuerza electoral, lo que sugiere que la movilización excedió estrictamente el campo político.

Las demandas que apuntan al gobierno y al sistema político sumaron 24,4\%; salariales y condiciones de trabajo 19,2\%; hábitat, infraestructura y transporte $10,9 \%$; justicia y derechos humanos $7 \%$; vivienda $6,2 \%$; educación $5,4 \%$; demandas sectoriales económicas $5 \%$; tierra $4,5 \%$; género y diversidad $4,1 \%$; medio ambiente $3,3 \%$; salud 2,2\%; y contra mega eventos deportivos $2,1 \%$. A diferencia del caso argentino, en Brasil fueron significativas las demandas contra el sistema político y por mejoras en infraestructura y transporte. De las tres principales demandas solo la salarial-condiciones de trabajo resultaba integrable a la luz de las políticas de salario mínimo implementadas por el PT. Aunque esta dispersión estadística permite afirmar la heterogeneización de las demandas, la preponderancia de la demanda contra el sistema político, conjuntamente con la respuesta del PT, fue decisiva para reforzar el carácter desafiante a la clase política en general, impugnador del modelo de desarrollo y no procesables por el sistema político de acuerdo a la definición de Germani (2003).

La diversificación del campo social en términos de los actores movilizados condujo a la reconfiguración de las relaciones entre el gobierno, los manifestantes y los partidos políticos de la oposición. Los sindicatos fueron los que se movilizaron un mayor cantidad de veces durante este periodo $(25,1 \%)$, siendo los sindicatos de la educación (20,4\%) los más activos, seguidos por los 
industriales (19,4\%); luego las organizaciones territoriales (15\%), estudiantes $(12,6 \%)$, organizaciones anticorrupción $(6,6 \%)$, agrupaciones ad hoc sin una estructura formal $(5,4 \%)$, vecinos $(5,3 \%)$, género y diversidad sexual $(5,2 \%)$, indígenas $(3,7 \%)$, partidarias $(3,3 \%)$, fuerzas de seguridad $(3,1 \%)$, trabajadores informales $(2,6 \%)$, colectividades de diverso tipo $(2,2 \%)$, ambientalistas $(2 \%)$ y derechos humanos (2\%).

Este proceso de heterogeneización fue, en parte, resultado de la estrategia de desmovilización, a saber, la participación en las instituciones estatales en lugar de la movilización en las calles. Esta fue implementada por el PT desde 2003, y profundizada a partir de 2011 cuando da Silva abrió mesas de diálogo y promovió cambios legislativos, generando condiciones de autonomía a los manifestantes y desmoralizante entre los aliados. Como resultado de esa estrategia el PT tuvo un repliegue significativo en la protesta callejera. Een el ciclo de 2013 emergieron nuevos colectivos como los ciberactivistas y los autoconvocados en línea (Tatagiba, 2019), quienes a pesar de contar con pocos recursos organizativos o conocimiento previo sobre cómo organizar una protesta masiva, fueron protagonistas de las protestas más trascendentales en la historia reciente de Brasil.

\subsection{Imaginarios antipolítica}

Estos cambios en la dinámica de la movilización influyeron en la reconfiguración de los imaginarios sociales dominantes que legitimaron las acciones del gobierno y del campo multiorganizacional. Uno de ellos fue el imaginario «liberal-punitivo» que no solo enmarcaba la acción colectiva, sino que también puso en discusión las formas en que las personas construyen su participación sociopolítica. La consolidación de este imaginario estuvo signada por dos eventos significativos: primero, Aécio Neves (PSDB) rechazó el resultado de las elecciones presidenciales de 2014 y convocó a protestas callejeras denunciando fraude electoral; segundo, los medios de comunicación empezaron a difundir información relacionada con la Operación Lava Jato. Este escándalo reflotaba el conocido Mensalão ocurrido en 2005 que llevó a la renuncia de asesores claves de da Silva. El caso de Lava Jato marcó un punto de inflexión en este tema ya que impregnó a la movilización social, contribuyendo a elaborar un imaginario antipolítica que no solo afectaba al gobierno, sino a todo el sistema político. A diferencia de los dos ciclos de protesta social anteriores —Diretas Já (1984) y Fora Collor (1992)_, en los que había un vínculo entre la movilización social y los imaginarios políticos 
expresados por el PT, esta vez no hubo ninguna conexión obvia entre las demandas planteadas por los actores movilizados y la oferta política existente.

Como se mencionó, la principal demanda 2011-2015 fue contra el gobierno y el sistema político, en una operación que homogeneizaba al PT con el resto de la clase política. Muchos de los que se sentían privados de sus derechos con el PT —organizaciones de estudiantes, territoriales, sectores medios movilizados por ciberactivistas y partidos políticos - aprovecharon la oportunidad para reclamar una nueva relación entre los movimientos sociales y el Estado. Algunos argumentos utilizados para justificar la acción colectiva, que hasta entonces permanecieron en los márgenes del debate público, como el apoyo a la dictadura, comenzaron a normalizarse. Tal fue el caso del Movimento Endireita Brasil (MEB), fundado en 2009 por Ricardo de Aquino Salles, actual Ministro de Medio Ambiente de Jair Bolsonaro.

No obstante, las demandas de los estudiantes, de género, indígenas, derechos humanos y medio ambiente también escalaron a niveles significativos para la historia de protestas en estos sectores en Brasil. ¿Hasta qué punto el giro a la izquierda habilitó el marco para la emergencia de nuevas demandas - como las laborales, de estudiantes, de género, entre otros- y hasta qué punto su impronta redistribucionista en lo económico supuso un límite a estas nuevas demandas? Lo significativo aquí es que las demandas liberal-punitivas coexistieron temporalmente con demandas por mayor protección del medio ambiente y expansión de derechos, dando a las primeras una plataforma de descontento transversal que terminó amplificando su impacto político.

\subsection{La crisis del largo ciclo de representación política}

La movilización social que surgió después del colapso financiero en Argentina y Brasil cambió los términos de referencia que definen la legitimidad - o autorización- de los actos políticos. En el caso de Brasil activó una crisis de un ciclo «largo» de representación política abierto con las luchas por la democratización, el nacimiento del PT y la reforma constitucional de 1988. En la práctica, el sistema político brasileño se caracteriza por ser un sistema de representación proporcional altamente fragmentado que favorece una forma particular de presidencialismo conocida como de coalición. Desde la promulgación de la Constitución de 1988, el partido mayoritario tuvo que formar coaliciones con otros partidos políticos para gobernar (Avritzer, 2019). A pesar de este problema de ingeniería institucional, la Constitución introdujo formas de participación directa que contribuyeron al 
surgimiento de experimentos verdaderamente participativos y democráticos que proporcionaron legitimidad al sistema. La participación directa fue alentada y canalizada a través de consejos consultivos que combinados con la representación política contribuyeron a construir un sistema de gobierno precario, pero relativamente estable. Da Silva expandió la política de los consejos al ámbito nacional como parte de su estrategia de desmovilización.

Cuando las multitudes salieron a las calles, el gobierno fue tomado por sorpresa y con poca imaginación para abordar las nuevas demandas. Si bien fue el Movimiento Pase Libre el que inicialmente lideró las protestas, fue el conservador Movimiento Brasil Libre el que se convirtió en uno de sus promotores ulteriores más destacados, planteando los problemas de corrupción sistémica como la demanda más importante y señalando al PT y al gobierno como responsables.

Dos factores interconectados adicionales contribuyeron a la crisis del sistema de representación establecido con el modelo de democratización. Ante el llamado a la reforma política exigido por algunas de las protestas de junio de 2013 Dilma intentó aprobar legislación que proponía el financiamiento público de las campañas electorales, pero la iniciativa fue

[344 ] derrotada, tensionando la relación con el Partido del Movimiento Democrático Brasileño (PMDB), principal socio en la coalición gubernamental. A medida que el PMDB se distanció del PT las elecciones de 2014 debilitaron aún más el margen de maniobra del gobierno, ya que el sistema electoral de lista abierta permitió que candidatos de la oposición fueran elegidos en la boleta del PT. La coalición gubernamental debilitada, la movilización histórica en las calles y el poder de los medios de comunicación de derecha resultaron en la producción de un golpe parlamentario; sin embargo, la oposición política había quedado igualmente desacreditada, creando una oportunidad para que partidos marginales como el Partido Social Liberal tomaran el centro del escenario y pusieran fin a un largo ciclo de representación política en Brasil.

\section{Conclusiones}

En pos de la comprensión de la crisis del giro a la izquierda en Argentina y Brasil a partir del análisis de la dinámica sociopolítica se siguió una perspectiva multidimensional, tratando de hacer inteligible la realidad sociopolítica mediante la reconstrucción de las características principales del ciclo de movilización, los imaginarios sociales en disputa y sus significados para la representación política. El argumento inicial indicaba que el ciclo 
surgido durante los gobiernos de Fernández de Kirchner y de Rousseff implicó una nueva dinámica sociopolítica, en tanto marcó el agotamiento de las fuentes de justificación que dieron legitimidad y potencia al giro a la izquierda, sin por ello construir un nuevo consenso societal. En este sentido, hay tres conclusiones principales interconectadas para resaltar:

En primer lugar, el ciclo de protestas 2012-2013 adoptó una forma integrada en Argentina y una no integrada en Brasil. Según el análisis de los datos, esto se debió a dos factores. El primero alude a la relación entre el campo de la movilización y el campo político. Por un lado, en ambos países se observa un componente emocional, antikirchnerista y anti-PT. En el caso argentino, la construcción de un colectivo más amplio encontró un lugar en la oposición política, contribuyendo a crear una serie de interacciones que resultaron en la victoria electoral de Cambiemos en 2015. Por el contrario, las protestas en Brasil enfrentaron al gobierno y al régimen político en su conjunto, expresando un descontento generalizado de los colectivos movilizados con el PT y los partidos de la oposición, lo que develó, al mismo tiempo, la ausencia de una racionalidad estratégica clara en el futuro. El segundo factor se refiere a las características que asumió el ciclo en cada país, relacionado con las tradiciones locales y los vínculos internos de las coaliciones de gobierno. Respecto de estos últimos, la vigencia de una gramática movimentista en Argentina implicaba relaciones de contigüidad entre la movilización y la política. El kirchnerismo no perdió la calle, solo su monopolio, de ahí que los sectores movilizados fueran antikirchneristas y kirchneristas; asimismo, la presencia de los exkirchneristas fue fundamental para evitar cuestionamientos a todo el ciclo de los gobiernos kirchneristas para concentrarse simplemente en el último de Fernández de Kirchner. En Brasil, la vigencia de una democracia participativa junto a la estrategia de desmovilización fue decisiva para que el PT no solo perdiera la calle, sino que entre los actores movilizados tampoco contara con aliados.

La segunda conclusión remite a los imaginarios. Si el giro a la izquierda significó una apuesta por la mayor inclusión social e igualdad como forma para superar la desigualdad y pobreza producidas por el neoliberalismo, la crisis de ese giro implicó tanto la activación de imaginarios asociados a la libertad y seguridad — considerados bajo amenaza_, y el reclamo porque el Estado no lograba garantizar mejoras sociales en un contexto de alto crecimiento económico. Respecto de los imaginarios de libertad y seguridad es llamativo el uso en ambos países del pánico moral a través de la idea de la «chavización», es decir, para marcar los peligros que podría traer la 
excesiva intervención del Estado, poniendo como límite la experiencia de la República Bolivariana de Venezuela. Este pánico moral funcionó como un motivo clave para movilizar a sectores que en general no tenían experiencia política. En ambos países, tanto el kirchnerismo como el lulismo se asociaron al populismo, representando la corrupción frente a la República.

A su vez, en Argentina había otro imaginario emergente: la creencia entre los miembros kirchneristas de que Cristina Fernández de Kirchner ya no representaba la pluralidad original del espacio kirchnerista. Esta heterogeneidad de los actores involucrados paradójicamente le jugó a favor en la cuenta final, en tanto le permitió consolidar una base de apoyo más acotada, pero al mismo tiempo más orgánica. En cambio, en Brasil el PT cerró filas contra los grupos movilizados y profundizó el grado de polarización con el imaginario liberal-punitivo emergente, desconociendo las demandas de aquellos que podían pedir por infraestructura o demandas de género, pero que no necesariamente objetaban el desempeño global del gobierno. En este sentido, si el kirchnerismo se mantuvo como un espacio propio - de menor cobertura, pero específico al fin-, el PT quedó subsumido dentro de la clase política en una operación discursiva que los volvió parte del problema y «del sistema». Por lo tanto, el desarrollo de un imaginario anti-PT significó al mismo tiempo la generación de un momento antipolítica como en la Argentina de 2001, en lugar que la de 2015, cuya demanda era iQue se vayan ellos!, y no iQué se vayan todos!

La tercera conclusión alude a la dimensión de la representación política y sus crisis en diferentes temporalidades. Por un lado, una de las consecuencias del ciclo de movilización en Argentina ha sido la emergencia de Cambiemos, una coalición opositora de centro-derecha. Aunque sus principales fuerzas políticas —Propuesta Republicana (PRO), Unión Cívica Radical (UCR) y Coalición Cívica- ya tenían existencia, la movilización propició su asociación, nacionalizó su discurso y le permitió ganar fuerza electoral. Vale recordar que sus consignas durante la campaña de 2015 fueron la lucha contra el hambre, contra el narcotráfico, la posibilidad de vivir mejor, mientras Macri garantizaba que ningún ciudadano iba a perder los derechos adquiridos durante el kirchnerismo. En este sentido, usando la metáfora de los juegos, por lo menos en la coyuntura electoral, se trató de un jugador insider que reivindicaba las mejoras sociales realizadas que proponía otro horizonte. El caso de Brasil fue diferente en tanto se rompió la alianza entre el PT y el PMDB, y aunque este último lideró el proceso de impeachment a Dilma Rousseff no logró evadir el descrédito generalizado que cubrió a la 
clase política tradicional, generando las condiciones para la emergencia de un outsider antipolítica, como el excapitán del Ejército, Jair Messias Bolsonaro.

Estos elementos son los que llevan a concluir que en términos de representación política hubo diferentes tipos de crisis. En Argentina, hubo una crisis con capacidad de resolverse dentro del sistema político, mientras en Brasil se recurrió a mecanismos de dudosa legalidad, lo que explica por qué hubo un golpe institucional en este último y una alternancia política de poder en el primero. La gramática movimentista establecida por el kirchnerismo demostró ser más resistente que la estrategia de desmovilización impulsada por el lulismo. El kirchnerismo estableció un estilo de gobernanza que articuló una amplia coalición multisectorial en el ámbito institucional, pero también construyó una sólida base social de apoyo con alianzas estratégicas con los sindicatos, el sector informal y los movimientos sociales. En este sentido, expresó un nuevo modo de representación política luego de la crisis de 2001. Por el contrario, el PT continuó la tradición política establecida en la propia Constitución nacional, apoyando la participación dentro de los espacios institucionales. Si bien esta estrategia resultó exitosa para establecer un consenso político democrático-participativo que sacó a millones de la pobreza, también burocratizó al PT hasta el punto de perder una conexión vital con su base social y el control de la calle. En este sentido, el PT representó la llegada de una «vieja» experiencia política originada en la resistencia a la dictadura. Su crisis simboliza también la crisis del largo ciclo de representación abierto con la transición a la democracia.

En últimas, los desafíos para la regeneración de alternativas políticas inclusivas que sobrevengan a «la crisis del giro a la izquierda» son más profundas en el caso de Brasil. La victoria del Frente de Todos en 2019 en primera vuelta electoral en Argentina apoya este argumento.

\section{Referencias bibliográficas}

1. Avritzer, Leonardo. (2019). The Double Crisis of Representation and Participation in Brazil. Representation, Journal of Representative Democracy, 55 (3), pp. 251-263. https://doi.org/10.1080/00344893.2019.1572646

2. Baczko, Bronislaw. (1984). Memorias y esperanzas colectivas. París: Payot.

3. Banaszak, Lee Ann. (2005). Inside and Outside the State: Movement Insider Status, Tactics, and Public Policy Achievements. In: Meyer, David S.; Jenness, Valerie \& Ingram, Helen (Eds.). Routing the Opposition: Social Movements, Public Policy, and Democracy (pp. 149-176). Minneapolis: University of Minnesota. 
4. Boltanski, Luc. (2014). De la crítica. Compendio de Sociología de la emancipación. Madrid: Akal. https://doi.org/10.4000/books.res.171

5. Cefaï, Daniel. (2011). Diez propuestas para el estudio de las movilizaciones colectivas. De la experiencia al compromiso. Revista de Sociología, 26, pp. 137-166. https://doi.org/10.5354/0719-529X.2011.27491

6. Cheresky, Isidoro. (2006). Ciudadanía, sociedad civil y participación política. Buenos Aires: Miño y Dávila.

7. De la Torre, Carlos. (2013). In the Name of the People: Democratization, Popular Organizations, and Populism in Venezuela, Bolivia, and Ecuador. European Review of Latin America and Caribbean Studies, 95, pp. 27-48. https://doi.org/10.18352/ erlacs.9229

8. Di Tella, Torcuato. (1998). Los partidos políticos: Teoría y análisis comparativo. Buenos Aires: A-Z.

9. Etchemendy, Sebastián. (2019). The Politics of Popular Coalitions: Unions and Territorial Social Movements in Post-Neoliberal Latin America (2000-15). Journal of Latin American Studies, 52 (1), pp. 157-188. https://doi.org/10.1017/ S0022216X19001007

10. Ferrero, Juan Pablo. (2014). Democracy against Neoliberalism in Argentina and Brazil: A Move to the Left. New York. Basingstoke: Palgrave Macmillan. https:// doi.org/10.1057/9781137395023

11. Ferrero, Juan Pablo. (2016). Post-Neoliberal Protest in Latin America as

[348 ] Struggle over the Name of «The People». Journal of Political Ideologies, 22 (1), pp. 52-73. https://doi.org/10.1080/13569317.2016.1255466

12. Ferrero, Juan Pablo; Natalucci, Ana \& Tatagiba, Luciana (Eds.) (2019). SocioPolitical Dynamics within the Crisis of the Left Turn in Argentina and Brazil. London: Rowman \& Littlefield International.

13. Ferrero, Juan Pablo; Tatagiba, Luciana \& Natalucci, Ana. (2018). The End of the Left Turn in Latin America? Dataset on Social Protest in Argentina and Brazil 20112016. Bath: University of Bath.

14. Germani, Gino. (2003). Autoritarismo, fascismos y populismo nacional. Buenos Aires: Temas.

15. Gold, Tomás. (2019). Tracing the Left Turn Crisis through Argentine Protest: The Anti-Kirchnerist Cycle of Mobilization (2012-2013). In: Ferrero, Juan Pablo; Natalucci, Ana \& Tatagiba, Luciana (Eds.). Socio-Political Dynamics within the Crisis of the Left: Argentina and Brazil (pp. 117-140). London: Rowman \& Littlefield International.

16. Levitsky, Steven \& Roberts, Kenneth. (2011). The Resurgence of the Latin American Left. Baltimore: Johns Hopkins University.

17. McAdam, Doug; Tarrow, Sidney y Tilly, Charles. (2005). Dinámica de la contienda política. Barcelona: Hacer. 
18. Natalucci, Ana. (2018). Entre la democratización y la república. Revisitando el ciclo de movilización en el último gobierno Kirchnerista (Argentina, 2012-2015). Estudios de Derecho, 75 (166), pp. 30-50. 10.17533/udea.esde.v75n166a02

19. Natalucci, Ana. (2019). Social Mobilisation and Politics in Argentina: Peak and Crisis of the Left Turn. In: Ferrero, Juan Pablo; Natalucci, Ana \& Tatagiba, Luciana (Eds.). Socio-Political Dynamics within the Crisis of the Left: Argentina and Brazil (pp. 65-94). London: Rowman \& Littlefield International.

20. Pereyra, Sebastián. (2013). Política y transparencia: la corrupción como problema público. Buenos Aires: Siglo xxı.

21. Pitkin, Hanna. (1985). El concepto de representación. Madrid: Centro de Estudios Constitucionales.

22. Rey, Julieta. (2019). What about Women During the Left Turn? The Case of \#NiUnaMenos in Argentina. In: Ferrero, Juan Pablo; Natalucci, Ana \& Tatagiba, Luciana (Eds.). Socio-Political Dynamics within the Crisis of the Left: Argentina and Brazil (pp. 243-260). London: Rowman \& Littlefield International.

23. Saad-Filho, Alfredo. (2013). Mass Protests under «Left Neoliberalism»: Brazil, June-July 2013. Critical Sociology, 39 (5), pp. 657-669. https/doi. org/10.1177/0896920513501906

24. Sidicaro, Ricardo. (2011). El partido peronista y los gobiernos kirchneristas. Nueva Sociedad, 234, pp. 74-94.

25. Silva, Eduardo. (2017). Reorganizing Popular Sector Incorporation: Propositions from Bolivia, Ecuador, and Venezuela. Politics and Society, 45 (1), pp. 91-122. 10.1177/0032329216683166

26. Singer, André. (2012). Os sentidos do lulismo: reforma gradual e pacto conservador. São Paulo: Companhia das Letras.

27. Stefanoni, Andrés. (2016). ¿Alba o crepúsculo? Geografías y tensiones del socialismo del siglo xxı. En: Rodríguez, Martín (ed.). ¿Por qué retrocede la izquierda? (pp. 81-117). Buenos Aires: Capital Intelectual.

28. Svampa, Maristella. (2013). «Consenso de los Commodities» y lenguajes de valoración en América Latina. Nueva Sociedad, 244, pp. 30-46.

29. Tarrow, Sidney. (1997). El poder en movimiento. Madrid: Alianza.

30. Tatagiba, Luciana. (2019). Crossroads of Brazilian Democracy: Dynamics of Social Mobilization During the Left Turn Cycle. In: Ferrero, Juan Pablo; Natalucci, Ana \& Tatagiba, Luciana (Eds.). Socio-Political Dynamics within the Crisis of the Left: Argentina and Brazil (pp. 37-64). London: Rowman \& Littlefield International.

31. Torre, Juan Carlos. (2017). Los huérfanos de la política de partidos revisited. Revista SAAP, 11 (2), pp. 241-249.

32. Vommaro, Gabriel. (2017). La larga marcha de Cambiemos: la construcción silenciosa de un proyecto de poder. Buenos Aires: Siglo Xxı.

33. Zucco, Cesar. (2008). The President's «New» Constituency: Lula and the Pragmatic Vote in Brazil's 2006 Presidential Elections. Journal of Latin American Studies, 40 (1), pp. 29-49. https/doi.org/10.1017/S0022216X07003628 